\title{
Schader-Preis 2010 für Wolf Lepenies
}

Der Soziologe Wolf Lepenies erhält den Schader-Preis 2010. Mit dem Schader-Preis zeichnet die Schader-Stiftung Gesellschaftswissenschaftler und -wissenschaftlerinnen aus, die durch ihre wissenschaftliche Arbeit und ihr öffentliches Wirken wichtige Beiträge für die Lösung gesellschaftlicher Probleme geleistet haben.

Der Preisträger des Schader-Preises 2010, Prof. Dr. Dr. h. c. Wolf Lepenies, erfüllt diese Anforderungen in ganz besonderem Maße. Er ist einer der wenigen deutschen Wissenschaftler, die zwischen Wissenschaft und gesellschaftlicher Einmischung in Fragen der Zivilgesellschaft keine Trennung vornehmen. Er lässt sich nicht einer Kategorie zuordnen: Er ist Soziologe, Historiker, Wissenschaftler, aber auch ein begnadeter Essayist, er kombiniert Ernsthaftigkeit und Ironie, Sachverstand und Leichtigkeit, und er ist vor allem auch ein Vermittler zwischen verschiedenen Kulturen und Disziplinen.

Von 1986 bis 2001 war Wolf Lepenies Leiter des Wissenschaftskollegs zu Berlin und hat in dieser Funktion maßgeblich dazu beigetragen, dass Menschen aus unterschiedlichen Disziplinen und Regionen der Welt in einen Dialog treten und gegenseitig voneinander lernen konnten. Das in Berlin erprobte Modell eines interkulturellen und interdisziplinären Austausches hat Wolf Lepenies nach 1989 in andere Länder getragen: So war er als Initiator am Aufbau von Kollegs in vielen anderen Staaten beteiligt wie z. B. in Ungarn, Rumänien und Mali. Wolf Lepenies ist ein „handelnder Intellektueller“ - ein Soziologe, der für seine wissenschaftlichen Arbeiten viele Auszeichnungen erhalten hat und für seine „Einmischung“ unter anderem mit dem Theodor-Heuss-Preis (2000) und dem Friedenspreis des Deutschen Buchhandels (2006) geehrt wurde.

Der Schader-Preis wird jährlich von der Schader-Stiftung verliehen und ist mit 15.000 Euro dotiert. Preisgericht ist das Kuratorium der Stiftung. Zu den bisherigen Preisträgern zählen unter anderem Lord Ralf Dahrendorf (2009), Klaus von Beyme (2008), FranzXaver Kaufmann (2007) und Gesine Schwan (2006). Die Darmstädter Schader-Stiftung fördert die Gesellschaftswissenschaften und deren Dialog mit der Praxis durch eigene Projekte oder Vorhaben, die sie mit anderen Einrichtungen im Netzwerk betreibt.

(C) VS-Verlag 2010 